\title{
HARDWARE EFFICIENT DESIGN OF FILTER BANKS FOR VIDEO CODING
}

\author{
Po-Cheng Wu, Liang-Gee Chen, Yuan-Chen Liu, and Yeong-Kang Lai \\ DSP/IC Design Lab., Department of Electrical Engineering \\ National Taiwan University \\ Taipei, Taiwan, R.O.C. China \\ E-mail: \{pcwu,lgchen,liu,lai\}@video.ee.ntu.edu.tw
}

\begin{abstract}
Since three-dimensional (3-D) subband coding has been introduced, most researches on $3-\mathrm{D}$ subband coding perform temporal filtering first. In this paper, we investigate the best permutation strategy for temporal, vertical, and horizontal filtering to minimize the requirement of delay elements and find that the results are opposite to our expectation.
\end{abstract}

\section{INTRODUCTION}

Recently, there has been rapid progress in the area of multirate digital signal processing. Applications of multirate systems include subband coding of video and audio signals, fast transforms using digital filter banks, wavelet analysis, and many others [1]-[3]. One of the most important applications of multirate systems is subband coding (SBC). Since it was introduced by Crochiere et al. [4] in 1976, subband coding has been an effective coding approach for video and audio applications [5]-[7]. Subband coding employs a filter bank for splitting the input signal, so the filter banks are essential to subband coding. Because filter banks usually deal with a large number of data, high speed computing hardware is indispensable for subband coding systems.

Subband coding was first extended to three dimensional (temporal, vertical, and horizontal) filtering, i.e., 3-D subband coding, by Karlsson and Vetterli [8]. Since then, most researches which deal with 3-D subband coding perform temporal filtering first, e.g., [8][13]. Since 3-D subband coding requires a large number of delay elements to store the intermediate data, delay elements dominate the hardware cost in this coding scheme. Therefore, the most important task remains how to minimize the requirement of delay elements. In this paper, we investigate the best permutation strategy for temporal, vertical, and horizortal filtering in different conditions in order to minimize the required number of delay elements.

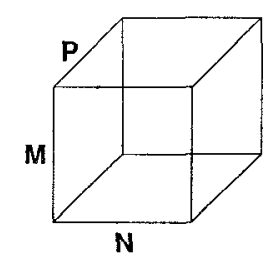

(a)

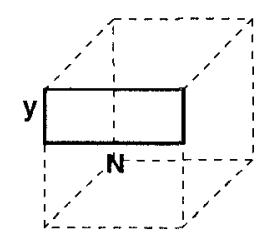

(c)

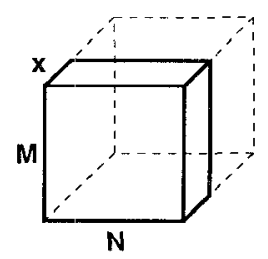

(b)

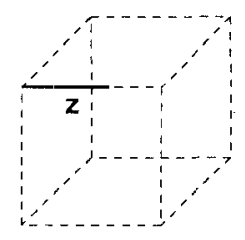

(d)
Figure 1: (a) Video format: $\mathbf{M}$ lines $\times \mathbf{N}$ pixels $\times \mathbf{P}$ frames/sec. (b) The required delay elements for temporal filtering. (c) The required delay elements for vertical filtering. (d) The required delay elements for horizontal filtering.

\section{DESIGN STRATEGY}

Let us assume the video format is $M$ lines $\times N$ pixels $\times$ $\mathbf{P}$ frames/sec as shown in Fig. 1(a), and the numbers of filter taps for temporal, vertical, and horizontal filtering are $x+1, y+1$, and $z+1$, respectively. Because the video data is read in line scan mode frame-by-frame, to perform filtering, the temporal filter needs to store the previous $x$ frames; the vertical filter needs to store the previous $y$ lines; the horizontal filter needs to store the previous $\mathbf{z}$ pixels. Therefore, the required numbers of delay elements for temporal, vertical, and horizontal filtering are $\mathrm{MNx}, \mathrm{Ny}$, and $\mathrm{z}$ as shown in Fig. 1(b), (c), and (d), respectively. In subband coding, we usually employ the FIR direct form filter. The advantage of employing the FIR direct form is that the lowpass 


\begin{tabular}{|c|c|c||c|c|c|c|}
\hline \multicolumn{2}{|c|}{ Permutations } & Stage $1(\times 1)$ & Stage 2 $(\times 2)$ & Stage 3 $(\times 4)$ & Total \\
\hline \hline $\mathbf{T}$ & $\mathbf{V}$ & $\mathbf{H}$ & $M N x$ & $N y$ & $z$ & $M N x+2 N y+4 z$ \\
\hline $\mathbf{T}$ & $\mathbf{H}$ & $\mathbf{V}$ & $M N x$ & $z$ & $N y / 2$ & $M N x+2 N y+2 z$ \\
\hline $\mathbf{V}$ & $\mathbf{T}$ & $\mathbf{H}$ & $N y$ & $M N x / 2$ & $z$ & $M N x+N y+4 z$ \\
\hline $\mathbf{V}$ & $\mathbf{H}$ & $\mathbf{T}$ & $N y$ & $z$ & $M N x / 4$ & $M N x+N y+2 z$ \\
\hline $\mathbf{H}$ & $\mathbf{T}$ & $\mathbf{V}$ & $z$ & $M N x / 2$ & $N y / 2$ & $M N x+2 N y+z$ \\
\hline $\mathbf{H}$ & $\mathbf{V}$ & $\mathbf{T}$ & $z$ & $N y / 2$ & $M N x / 4$ & $M N \mathbf{x}+\mathbf{N y}+\mathbf{z}$ \\
\hline
\end{tabular}

Table 1: Comparison of the required delay elements for different filtering permutations in 3-D subband coding with eight-full bands.

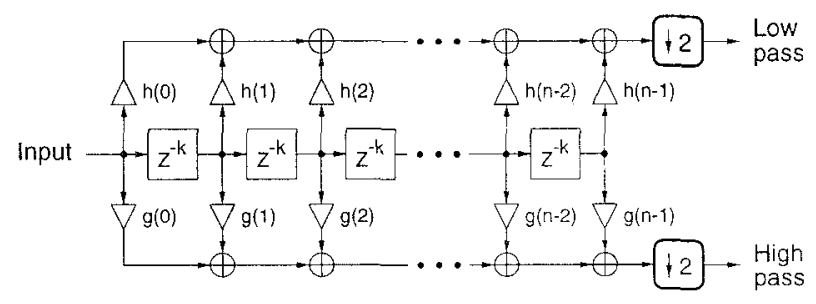

Figure 2: FIR direct form filter for subband coding.

filter and the highpass filter can share the same delay elements as shown in Fig. 2.

\subsection{Eight-Full Bands}

We will first consider 3-D subband coding with eightfull bands as shown in Fig. 3. From Fig. 3, we find that by employing the FIR direct form filters, stage 1 requires one set of delay elements; stage 2 requires two sets of delay elements; stage 3 requires four sets of delay elements. Because there are decimation operations performed along the filtering direction after filtering in each stage, the output data at each branch is half the input data. Therefore, the delay elements required in each stage are different and depend on the different filtering permutations. Fig. 4 gives two examples of the required delay elements for different filtering permutations: (a) temporal, vertical, followed by horizontal filtering; (b) horizontal, vertical, followed by temporal filtering. Table 1 lists the required numbers of delay elements for six different permutations of temporal, vertical, and horizontal filtering. Note that, because we use the separable FIR filters, the filtering results are identical even though the filtering permutations are $d-$ ifferent. From Table 1, we find that for eight-full-band splitting, the cascade strategy which requires the minimum number of delay elements is horizontal, vertical, followed by temporal filtering, i.e., $(\mathrm{H}, \mathrm{V}, \mathrm{T})$.

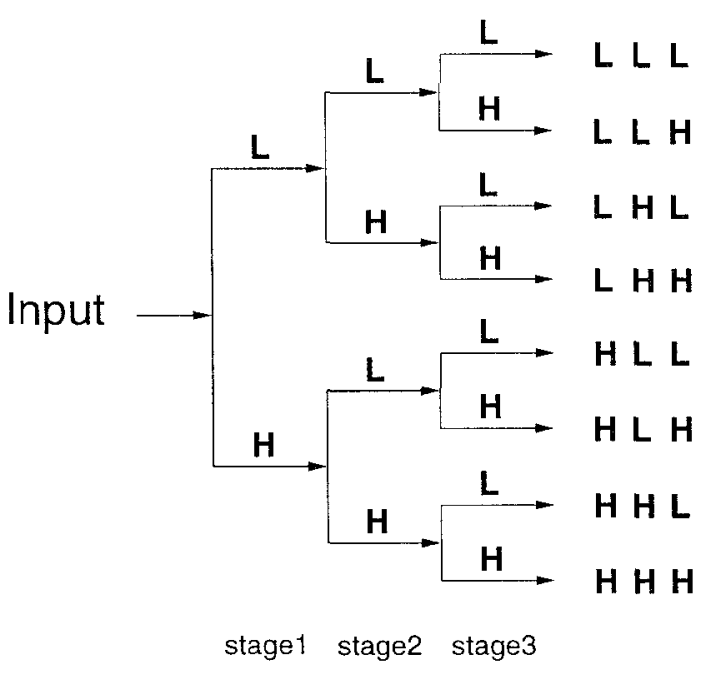

Figure 3: 3-D subband coding with eight-full bands.
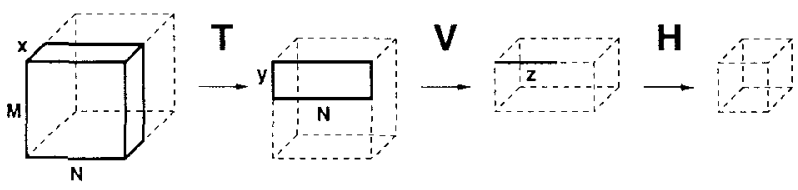

(a)
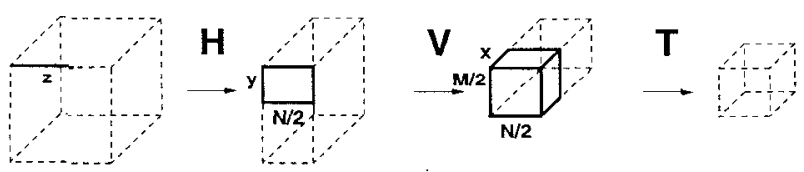

(b)

Figure 4: The required delay elements for: (a) temporal, vertical, followed by horizontal filtering; (b) horizontal, vertical, followed by temporal filtering. 


\begin{tabular}{|c|c|c||c|c|c|c|}
\hline \multicolumn{2}{|c|}{ Permutations } & Stage 1 $(\times 1)$ & Stage 2 $(\times 2)$ & Stage 3 $(\times 3)$ & Total \\
\hline \hline $\mathbf{T}$ & $\mathbf{V}$ & $\mathbf{H}$ & $M N x$ & $N y$ & $z$ & $M N x+2 N y+3 z$ \\
\hline $\mathbf{T}$ & $\mathbf{H}$ & $\mathbf{V}$ & $M N x$ & $z$ & $N y / 2$ & $M N x+3 N y / 2+2 z$ \\
\hline $\mathbf{V}$ & $\mathbf{T}$ & $\mathbf{H}$ & $N y$ & $M N x / 2$ & $z$ & $M N x+N y+3 z$ \\
\hline $\mathrm{V}$ & $\mathbf{H}$ & $\mathbf{T}$ & $N y$ & $z$ & $M N x / 4$ & $3 M N x / 4+N y+2 z$ \\
\hline $\mathbf{H}$ & $\mathbf{T}$ & $\mathbf{V}$ & $z$ & $M N x / 2$ & $N y / 2$ & $M N x+3 N y / 2+z$ \\
\hline $\mathbf{H}$ & $\mathbf{V}$ & $\mathbf{T}$ & $z$ & $N y / 2$ & $M N x / 4$ & $\mathbf{3 M N x} / 4+\mathbf{N y}+\mathbf{z}$ \\
\hline
\end{tabular}

Table 2: Comparison of the required delay elements for different filtering permutations in 3-D subband coding with four-reduced bands.

\begin{tabular}{|c|c|c||c|c|c|c|}
\hline \multicolumn{2}{|c|}{ Permutations } & Stage 1 $(\times 1)$ & Stage 2( $\times 2)$ & Stage 3 $(\times 3)$ & Total \\
\hline \hline $\mathbf{T}$ & $\mathbf{V}$ & $\mathbf{H}$ & $M N x a$ & $N y b$ & $z c$ & $M N x a+2 N y b+3 z c$ \\
\hline $\mathbf{T}$ & $\mathbf{H}$ & $\mathbf{V}$ & $M N x a$ & $z b$ & $N y c / 2$ & $M N x a+3 N y c / 2+2 z b$ \\
\hline $\mathbf{V}$ & $\mathbf{T}$ & $\mathbf{H}$ & $N y a$ & $M N x b / 2$ & $z c$ & $M N x b+N y a+3 z c$ \\
\hline $\mathbf{V}$ & $\mathbf{H}$ & $\mathbf{T}$ & $N y a$ & $z b$ & $M N x c / 4$ & $3 M N x c / 4+N y a+2 z b$ \\
\hline $\mathbf{H}$ & $\mathbf{T}$ & $\mathbf{V}$ & $z a$ & $M N x b / 2$ & $N y c / 2$ & $M N x b+3 N y c / 2+z a$ \\
\hline $\mathbf{H}$ & $\mathbf{V}$ & $\mathbf{T}$ & $z a$ & $N y b / 2$ & $M N x c / 4$ & $3 M N x c / 4+N y b+z a$ \\
\hline
\end{tabular}

Table 3: Comparison of the required delay elements for different filtering permutations considering the finite wordlength effects in 3-D subband coding with four-reduced bands. (The unit in this table is bits.)

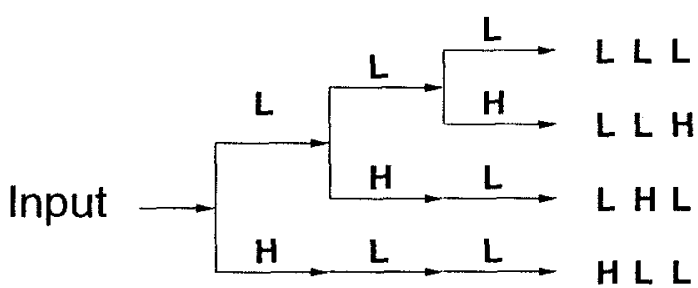

stage1 stage2 stage3

Figure 5: 3-D subband coding with four-reduced bands.

\subsection{Four-Reduced Bands}

In order to achieve a high compression ratio in $3-D$ subband coding, we often retain the four lower bands and discard the higher bands as shown in Fig. 5. This is wavelet-like splitting. Thus, from Fig: 5, we find that stage 1 requires one set of delay elements; stage 2 requires two sets of delay elements; stage 3 requires three sets of delay elements. The required numbers of delay elements for different permutations are listed in Table 2. From Table 2, we find that for four-reducedband splitting, the cascade strategy which minimizes the delay elements is horizontal, vertical, followed by temporal filtering, i.e., $(\mathbf{H}, \mathrm{V}, \mathbf{T})$.

\subsection{Finite Wordlength Effects}

Finally, if we further consider the finite wordlength effects to enhance the accuracy of subband coding, we assume that the wordlengths of delay elements in stage 1 , stage 2 , and stage 3 are $\mathbf{a}, \mathbf{b}$, and $\mathbf{c}$ bits, respectively. The relation between $\mathbf{a}, \mathbf{b}$, and $\mathbf{c}$ is usually $\mathbf{a} \leq \mathbf{b} \leq \mathbf{c}$. Now, we still look at $3-\mathrm{D}$ subband coding with fourreduced bands. The required bits of delay elements for different permutations are listed in Table 3. From Table 3 , we are unable to distinguish which permutation strategy is the best choice. Therefore, we make the following assumptions: the video format is $M=512$ lines and $\mathrm{N}=1024$ pixels; the temporal filter has $\mathbf{x}+1=2$ taps (in order to minimize the delay time); the vertical filter has $y+1=8$ taps; the horizontal filter has $z+1=16$ taps; the wordlengths of delay elements in stage 1 , stage 2 , and stage 3 are $\mathbf{a}=8$ bits, $\mathbf{b}=9$ bits, and $\mathbf{c}=10$ bits, respectively. After careful calculation, we find that if we consider the finite wordlength effect$s$ in the above conditions, the cascade strategy which minimizes the delay elements is vertical, horizontal, followed by temporal filtering, i.e., $(\mathbf{V}, \mathbf{H}, \mathbf{T})$.

\section{CONCLUSIONS}

From above discussion, we find that the results are opposite to our expectation. Performing the temporal 
filtering first is not the best strategy. On the contrary, placing the temporal filter at final stage will save the delay elements. We also find that the best permutation strategy for temporal, vertical, and horizontal filtering depends on different conditions. Finally, for the synthesis filter banks or a higher number of filtering stages in 3-D subband/wavelet coding, by employing the same scheme, we can also derive the best design strategy which minimizes the requirement of delay elements.

\section{REFERENCES}

[1] R. E. Crochiere and L. R. Rabiner, Multirate Digital Signal Processing. Englewood Cliffs, NJ: Prentice Hall, 1983.

[2] P. P. Vaidyanathan, Multirate Systems and Filter Banks. Englewood Cliffs, NJ: Prentice Hall, 1993.

[3] N. J. Fliege, Multirate Digital Signal Processing. New York: Wiley, 1994.

[4] R. E. Crochiere, S. A. Webber, and J. L. Flanagan, "Digital coding of speech in subbands," Bell Syst. Tech. J., vol. 55, pp. 1069-1085, Oct. 1976.

[5] J. W. Woods and S. D. O'Neil, "Subband coding of images," IEEE Trans. Acoust., Speech, Signal Processing, vol. ASSP-34, pp. 1278-1288, Oct. 1986.

[6] H. Gharavi, "Subband coding algorithms for video applications: videophone to HDTV-conferencing," IEEE Trans. Circuits Syst. Video Technol., vol. 1, no. 2, pp. 174-183, June 1991.

[7] E. B. Richardson and N. S. Jayant, "Subband coding with adaptive prediction for $56 \mathrm{kbits} / \mathrm{s}$ audio," IEEE Trans. Acoust., Speech, Signal Processing, vol. ASSP-34, pp. 691-696, Aug. 1986.

[8] G. Karlsson and M. Vetterli, "Three dimensional sub-band coding of video," in Proc. IEEE Int. Conf. Acoust., Speech, Signal Processing, 1988, pp. 1100-1103.

[9] J. Hartung, "Architecture for real-time implementation of three-dimensional subband video coding," in Proc. IEEE Int. Symp. Circuits Syst., vol. 3,1992 , pp. 225-228.

[10] K. N. Ngan and W. L. Chooi, "Very low bit rate video coding using $3 \mathrm{D}$ subband approach," IEEE Trans. Circuits Syst. Video Technol., vol.4, no. 3, pp. 309-316, June 1994.
[11] C. I. Podilchuk, N. S. Jayant, and N. Farvard$\mathrm{m}$, "Three-dimensional subband coding of video," IEEE Trans. Image Processing, vol. 4, no. 2, pp. 125-139, Feb. 1995.

[12] F. Fan, S. Simon, I. Bruyland, W. Zhu, B. D. Canne, and M. V. Bladel, "A method for hierarchical subband HDTV splitting," IEEE Trans. Circuits Syst. Video Technol., vol. 5, no. 3, pp. 225-230, June 1995.

[13] C. H. Chou and C. W. Chen, "A perceptually optimized 3-D subband codec for video communication over wireless channels," IEEE Trans. Circuits Syst. Video Technol., vol. 6, no. 2, pp. 143-156, Apr. 1996. 